\title{
Integral System for Detecting Evaporation Residues in Fusion Reactions
}

\author{
O.A. Capurro, J.E. Testoni, G.V. Martí, A. Arazi, J.O. Fernández Niello, A.J. Pacheco, \\ Laboratorio TANDAR, Departamento de Física, Comisión Nacional de Energía Atómica, \\ Av. Gral. Paz 1499 (1650), Partido de Gral. San Martín, Buenos Aires, Argentina
}

M.E. Ortega, J. Tiffenberg, and M. Fleitas

Departamento de Física, Universidad de Buenos Aires, (1428) Buenos Aires, Argentina

Received on 1st November, 2004

\begin{abstract}
We have designed and constructed an integral system that involves two electrostatic deflectors followed by a small ionization chamber with a multi-wire-proportional-counter in its entrance. The function of the former part is to separate evaporation residues from beam ions using their different electric rigidities while the latter is to detect these evaporation residues being distinguished from the beam-like particles originated in slits. Up to now, both devices have been tested and evaluated performing different experiments: the double-electrostatic deflector through scattering studies and the ionization chamber by means of scattering and fusion reactions. We present a brief description of the two devices and some results obtained in different tests.
\end{abstract}

\section{INTRODUCTION}

One way to measure the fusion cross section is just to detect the nuclei resulting from the evaporation of the compound nucleus. These evaporation residues (ER) are emitted at forward angles and, therefore, they merge with the projectiles. Typical rates for projectiles and ER are $10^{11}$ and $10^{3}$ particles per second, respectively; therefore, it is essential to reduce the beam-like particle rate in the detector. Moreover, although this requirement could be achieved, a second difficulty is present in any fusion experiment: to differentiate the fusion products from the projectile-like particles originated by slit-scattering and/or multi-scattering in the target.

\section{ELECTROSTATIC DOUBLE-DEFLECTOR AND ITS PERFORMANCE}

The first problem pointed out in the previous Section has been answered employing recoil separators [1-3] but some limitations has to be kept in mind, e.g. low and uncertain efficiency and -in several cases- restricted angular range. Another possibility has been the use of electrostatic deflectors which can separate ER from projectiles thanks to the very different electric rigidity of ones and the others [4-8]. However, the evaporation residues have a wide range in energy and charge state; thus, in these devices, they are deflected differently and not all of them are finally observed. These features can be seen in Fig. 1a). Here, we show a simple deflector which has a grid as plate. The plotted trajectories were calculated for the system ${ }^{13} \mathrm{C}+{ }^{105} \mathrm{Pd}$ at $E_{l a b}=41 \mathrm{MeV}$. On the one hand, we can see that the beam is clearly separated from the fusion products. On the other hand, it is clear too that the evaporation residues are dispersed over a wide angular range. The two groups shown in the picture correspond to the lowest and the highest energy of the evaporation residues. The wide dispersion of the evaporation residues can be lowered employing a second electrostatic field -instead a magnetostatic field as in a Wien filter [9]-. Figure 1b) shows the predicted effect. The direction of the second field is just opposite to the first one. Its function is to correct the more deflected trajectories.

Several devices -employing different insulator materials and designs- were essayed for the double deflector trying to reach high voltages without sparks in the scattering chamber at a pressure of $\sim 10^{-5}$ Torr. From the literature [10] and our tests we can conclude that three practical considerations need to be taken into account to attain high voltages without reaching the electrical breakdown process: 1) The performance of a vacuum gap is much better than that of a solid insulator of similar dimensions; 2) It is important to have insulator lengths as long as it is possible; 3 ) The high voltage electrodes have to be mechanically polished and their edges properly rounded. Following these elemental concepts, our last design can reach electric fields of about $15 \mathrm{kVolts} / \mathrm{cm}$ without sparks; no previous conditioning is necessary. The anode plates are made of polished aluminium and the cathode grid consists of strained $\mathrm{Cu}-\mathrm{Be}$ wires $(\phi=50 \mu \mathrm{m})$. The length of the device is 31 $\mathrm{cm}$ and the distance between each plate and the grid is 3.25 $\mathrm{cm}$. The material used as solid insulator (Nylon) to hold up the electrodes was cleaned with Extran cleanser prior to the assembling.

In order to study the performance of the double deflector, we have carried out elastic scattering experiments employing the setup shown in the Fig. 2 where one can see a movable $\mathrm{Si}$ detector behind the double deflector in order to search the ions of interest. The ${ }^{12} \mathrm{C}+{ }^{n a t} \mathrm{Pd}$ system was studied at a bombarding energy of $30 \mathrm{MeV}$ to detect two kinds of ions: low mass with high energy (projectiles) and medium mass with low energy (recoils). The electrostatic separator was placed at $\sim 30^{\circ}$ respect to the incident beam and both types of ion enter into the deflector in a middle point between the first anode and the grounded grid passing through a circular collimator of $1 \mathrm{~mm}$ diameter. The expected angular separation between them can be observed in the Fig. 3 showing the angular distribution for each ion when an appropriated electric field is set between the plates and the grounded grid (voltages of 40 and 


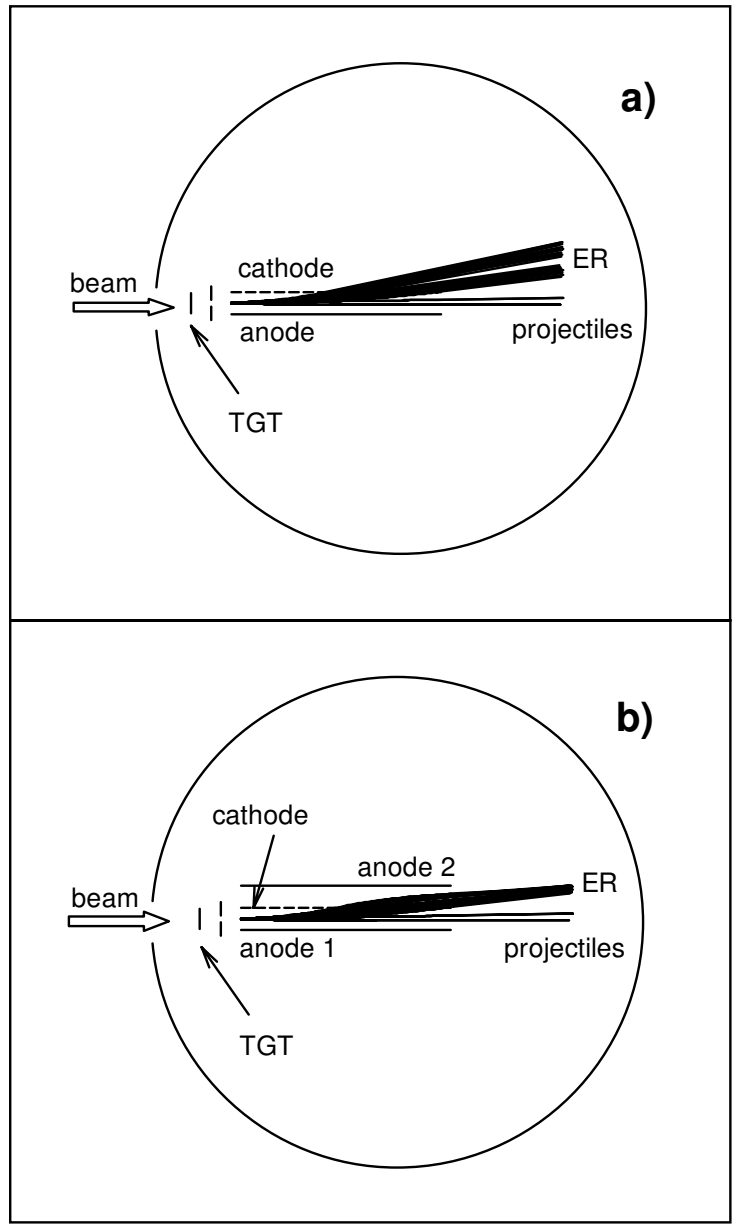

FIG. 1: Trajectories of projectiles and evaporation residues for the system ${ }^{13} \mathrm{C}+{ }^{105} \mathrm{Pd}$ at $E_{l a b}=41 \mathrm{MeV}$ in a simple a) and a double b) electrostatic deflector.

$30 \mathrm{kVolts}$ applied on the first and second anode, respectively). A step forward was to evaluate the influence of a $2 \mathrm{~mm}$ strip collimator placed close to the target with the aim of reducing the angular variations associated to the beam spot movement. In the upper part of the Fig. 4 we can observe that the FWHM of the angular spread corresponding to the carbon ions is reduced by a factor $\sim 2$. The lower part of the same figure shows how, using the strip collimator, carbon ions of $29 \mathrm{MeV}$ are displaced $\sim 1$ degree when an electric field of about $13 \mathrm{kVolts} / \mathrm{cm}$ is applied between the first plate and the grounded grid. The strip collimator allows a good separation of peaks with angular positions differing in one degree.

\section{MULTI-DETECTOR}

Although the double deflector is able to separate fusion products from the projectile ions, it is expected that an important number of projectile-like particles -resulting mainly from slit scattering- arrives at the detector. These ions play a crucial

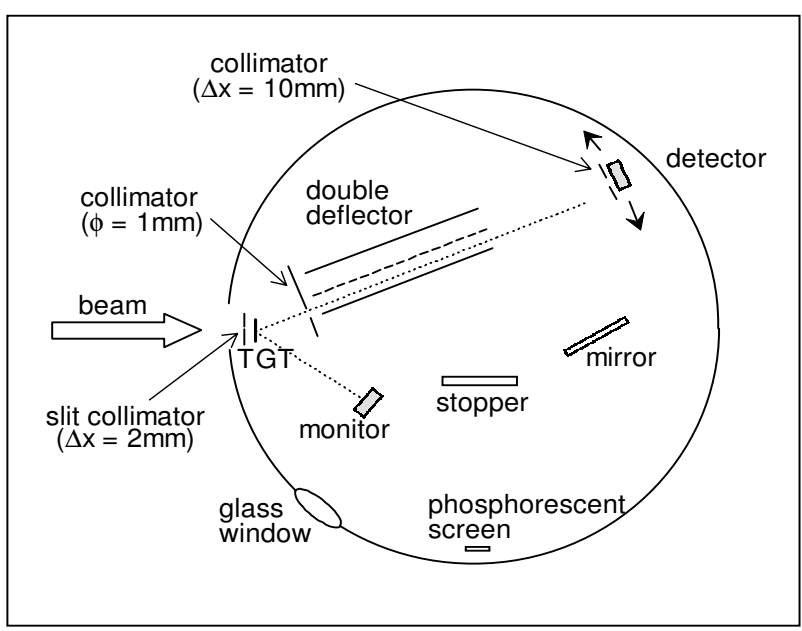

FIG. 2: Setup used in the elastic scattering experiments carried out in order to study the performance of the double deflector. Some elements associated to the focusing procedure are also displayed.

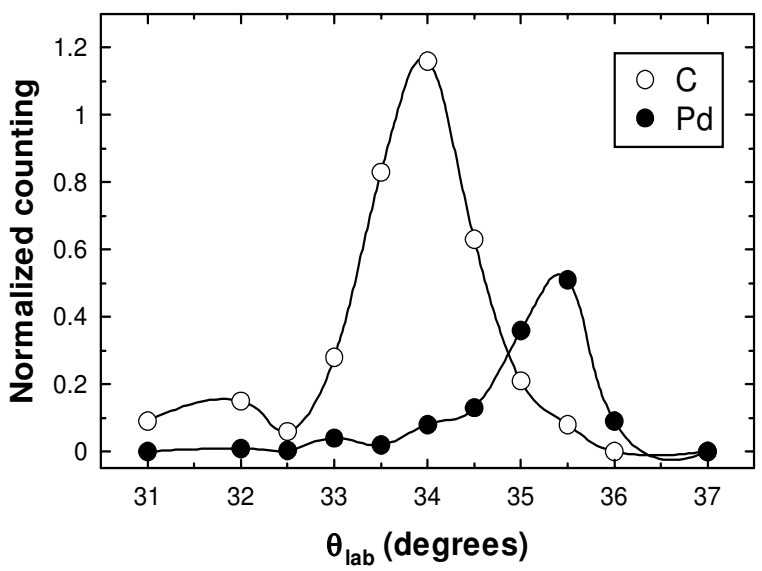

FIG. 3: Angular distributions of carbon and palladium ions corresponding to voltage differences of 40 and $30 \mathrm{kVolts}$ between the grounded grid and the first and the second anode, respectively.

role due to the similar energies of these particles compared to those corresponding to the ER ions. Therefore, typical $\Delta \mathrm{E}-\mathrm{E}$ telescopes are unsuitable to make out ones and the others resulting necessary to measure the time-of-flight of both type of ions.

We have devised and constructed a small detector, TOFICHE, that measure Time-Of-Flight, partial energy loss in an Ionization CHamber and residual Energy. A schematic drawing of this multi-detector can be seen in Fig. 5. Following a Mylar window, there is a multi-wire-proportionalcounter (MWPC) that consists of three circular grids $(\phi=40$ $\mathrm{mm}$ ) separated by $1.5 \mathrm{~mm}$ each other. This MWPC allows to generate a start signal for a time-of-flight measure. A typical ionization chamber permits to obtain a signal proportional to the energy loss in its gaseous region. A silicon detector provides an energy signal and a stop signal for the time-of-flight 


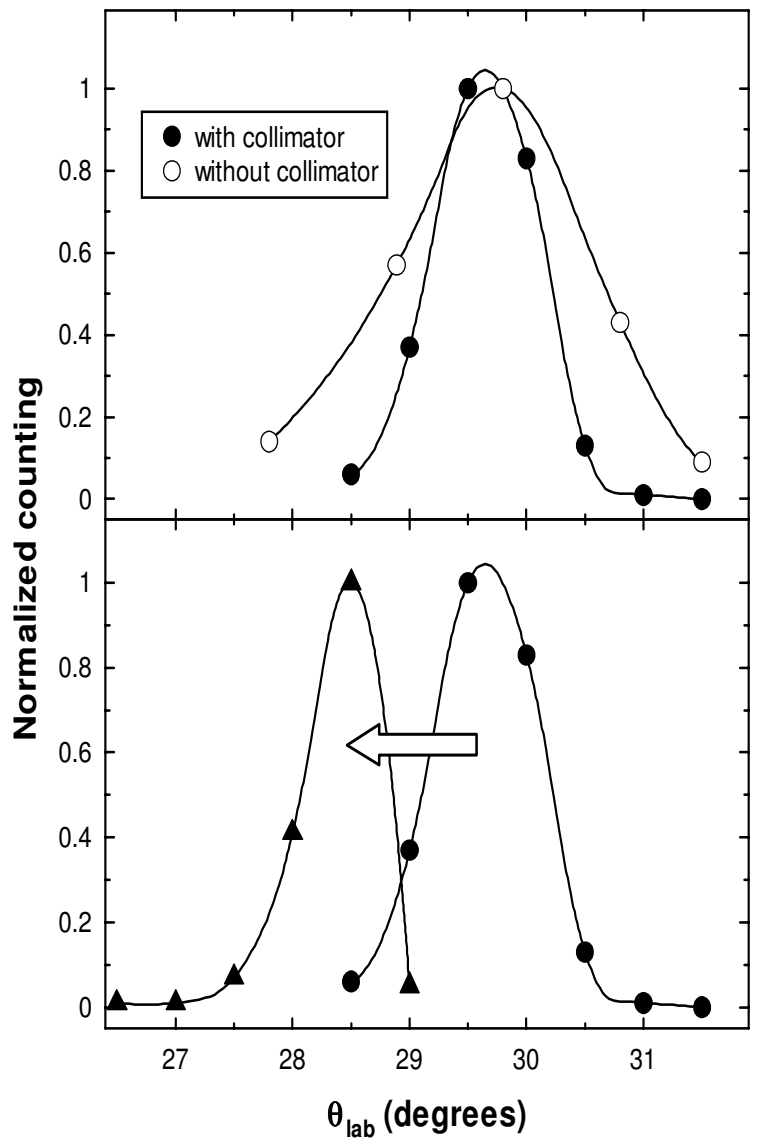

FIG. 4: The upper panel shows the reduction of the angular spread caused by the presence of the strip collimator. A satisfactory separation of one degree is achieved using this collimator as can be seen in the lower panel.

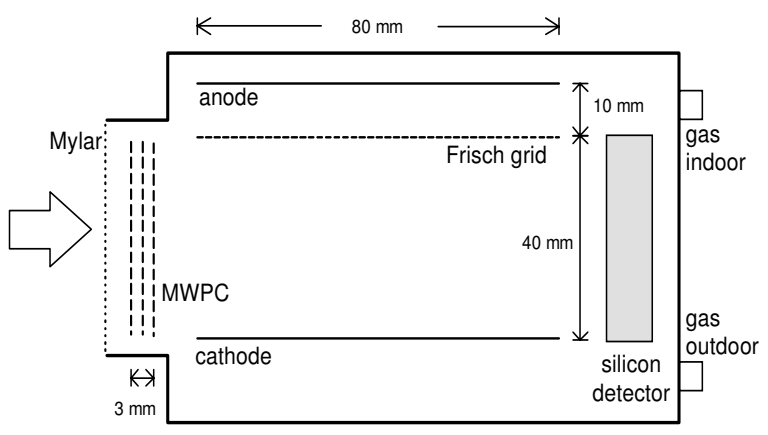

FIG. 5: Schematic representation of the multi-detector TOFICHE.

determination.

In order to characterize the multi-detector TOFICHE, a variety of systems were analyzed by elastic scattering experiments combining four projectiles $\left({ }^{12} \mathrm{C},{ }^{35} \mathrm{Cl},{ }^{80} \mathrm{Se}\right.$, and $\left.{ }^{127} \mathrm{I}\right)$ with two targets $\left({ }^{12} \mathrm{C}\right.$ and $\left.{ }^{197} \mathrm{Au}\right)$. Different values of partial loss of energy, residual energy, and time of flight were obtained for the projectile and recoil scattered ions changing the

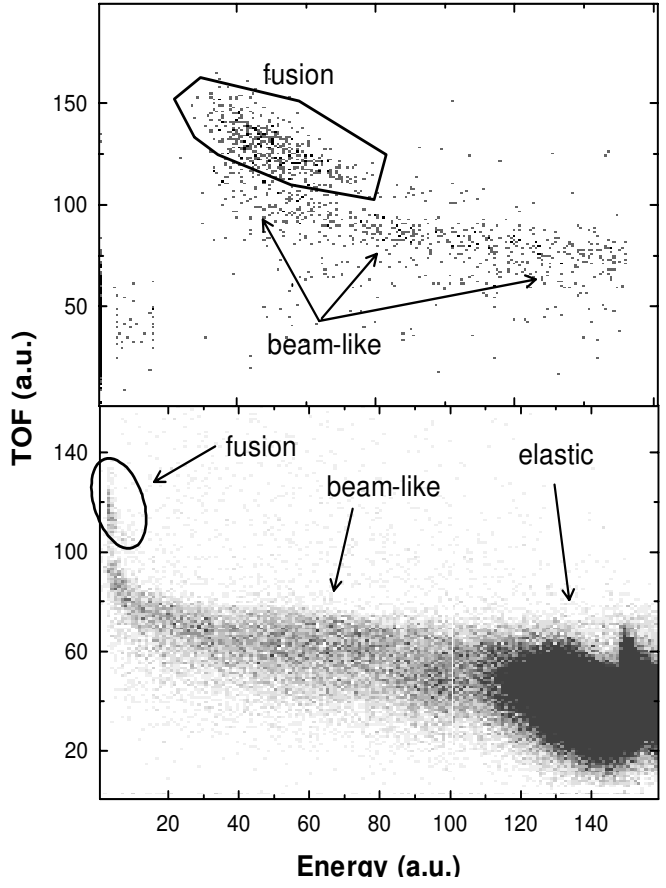

FIG. 6: Time-of-flight vs. residual energy spectra for the ${ }^{12} \mathrm{C}(54 \mathrm{MeV})+{ }^{105} \mathrm{Pd}$ system. In the upper panel a detailed plot of the fusion region is shown; in the lower panel, a panoramic picture of the spectrum is displayed.

detection angle. In spite of the good results obtained from these experiments, we believed that the best test of confidence would be given by analyzing a fusion reaction experiment. In order to do this, carbon ion beam delivered by the tandem accelerator of the TANDAR Laboratory in Buenos Aires bombarded an enriched metallic ${ }^{105} \mathrm{Pd}$ target. The irradiation was carried out at a bombarding energy of $54 \mathrm{MeV}(\sim 30 \%$ above the Coulomb barrier). The multi-detector TOFICHE was placed at $\theta_{l a b}=7.6^{\circ}$ and operated with isobutane at a pressure of 4 Torr. Time-of-flight vs. energy spectra obtained for the ${ }^{12} \mathrm{C}(54 \mathrm{MeV})+{ }^{105} \mathrm{Pd}$ system can be seen in the Fig. 6 . The upper panel of this figure shows clearly that the evaporation residues are differentiated from the projectile-like particles. The spectrum shown in the lower panel gives a panoramic view of the time-of-flight vs. energy plot that includes elastic, beamlike and fusion events. This picture reveals as indispensable the use of an electrostatic deflector in order to avoid that carbon ions, scattered by the target and slits, overwhelm the site where the evaporation residues must be registered.

\section{SUMMARY AND CONCLUSIONS}

A typical device employed in fusion reactions to separate evaporation residues from beam-like ions is an electrostatic deflector. In order to reduce the broad angular range of the former ions due to different energies and charge states, we have designed and constructed a system that involves two electros- 
tatic deflectors instead of a single one. To detect the evaporation residues we have devised and built a small ionization chamber from which one can obtain four signals: energy loss, residual energy and two timing signals (start and stop); these two latter signals let us to measure the time-of-flight. Thus, the measurements associated to the energy and the time of flight will allow us to distinguish the evaporation residues from the beam-like particles. Up to now, both devices have been tested and evaluated in several experiments: the double electrostatic deflector through scattering studies and the ionization chamber by means of scattering and fusion reactions.
The good results obtained from all these experiments give us reliance to affirm that both devices can be used in fusion cross section measurements. The natural next step will be try our integral system of detection as a whole.

\section{Acknowledgments}

Some of us (JET, JOFN, AJP) are fellows of the Carrera del Investigador Científico of the CONICET (Argentina).
[1] K. Subotic et al., Nucl. Instr. and Meth. A 481, 71 (2002)

[2] M. Leino, Nucl. Instr. and Meth. B 204, 129 (2003)

[3] C.N. Davids, Nucl. Instr. and Meth. B 204, 124 (2003)

[4] W.S. Freeman et al., Phys. Rev. Lett. 50, 1563 (1983); Phys. Rev. C 28, 919 (1983).

[5] M. Dahlinger et al., Nucl. Instr. and Meth. 219, 513 (1984)

[6] S. Beghini et al., Nucl. Instr. and Meth. A 239, 585 (1985)
[7] R. Schicker et al., Nucl. Instr. and Meth. A 269, 585 (1988)

[8] A. Charlop, Lecture Notes in Physics, Springer-Verlag, 317 (1988)

[9] J.X. Wei et al., Nucl. Instr. and Meth. A 306, 557 (1991)

[10] R.V. Latham, "High voltage vacuum insulation”, Academic Press, 1981. 